\title{
Neurophysiologic Studies and Cognitive Function in Congenital Hypothyroid Children
}

\author{
GIOVANNA WEBER, VIRGINIA SIRAGUSA, GIAN FILIPPO RONDANINI, LAURA MARIA \\ PRINA CERAI, STEFANO MORA, JOLE COLOMBINI, STEFANIA MEDAGLINI, CHIARA LIA, \\ TIZIANA LOCATELLI, GIANCARLO COMI, AND GIUSEPPE CHIUMELLO
}

Endocrine Unit, the Department of Pediatrics, and the Department of Neurology, Scientific Institute, $H$ San Raffaele, University of Milan, Milan, Italy \begin{abstract}
MBS
Minor neurologic and intellectual impairments have been
described in some congenital hypothyroid $(\mathrm{CH})$ children in spite of early detection by neonatal screening. The aim of our study was to assess cognitive functions as well as neurophysiologic parameters in hypothyroid children and to compare children detected by neonatal screening (group A) versus hypothyroid patients clinically diagnosed before the beginning of the screening program (group B). Group A consisted of 15 children (13 girls, mean age at the beginning of treatment $33 \mathrm{~d}$ ). Group $\mathrm{B}$ consisted of 11 patients ( 7 girls, mean age at the start of treatment $10.1 \mathrm{mo}$ ). Twenty age-matched healthy children were studied as a control group for neurophysiologic tests. Neurophysiologic tests (Auditory P 300, long latency somatosensory evoked potentials (LL-SEP) were performed along with IQ evaluation. Abnormalities of neurophysiologic tests were detected in $82 \%$ of clinically diagnosed hypothyroid children. Surprisingly, $47 \%$ of
\end{abstract}

Neonatal screening programs and subsequent early replacement treatment have been successful in reducing the most severe effects of $\mathrm{CH}$ on neuropsychologic development (1-6); however, early treated $\mathrm{CH}$ children with normal IQ may show slight psychomotor abnormalities, such as deficit in motor skills (particularly in relation to balance), fine motor disorders, clumsiness, learning difficulties, and behavior problems $(1,5)$. All of these disorders are suggestive of minimal brain damage.

Neurophysiologic studies on $\mathrm{CH}$ patients have been concerned primarily with stimulus-related potentials (short latency components) that analyze the integrity of the primary sensory pathways (7). The LL-SEP and the event-related potentials can give information about CNS stimulus processing and about the integrity of sensory information processing within the cerebral cortex (8). Only one group, however, reported results of short latency somatosensory evoked potential examinations in $\mathrm{CH}$ newborns (9).

Received February 8, 1994; accepted December 6, 1994

Correspondence and reprints requests: Stefano Mora, M.D., Clinica Pediatrica III, H San Raffaele, via Olgettina 60, 20132 Milan MI, Italy. the children detected by neonatal screening, having normal mental development index, showed at least one abnormal neurophysiologic test. LL-SEP latencies were found significantly increased in both groups of $\mathrm{CH}$ patients compared with controls. Our data are suggestive for a prenatal or perinatal CNS damage in some children with congenital hypothyroidism, despite early treatment. (Pediatr Res 37: 736-740, 1995)

$\quad$ Abbreviations
CH, congenital hypothyroidism
LL-SEP, long latency-somatosensory evoked potential
WISC-R, Wechsler Intelligence Scale for Children-Revised
$\mathbf{T}_{\mathbf{4}}$, thyroxine
ANOVA, analysis of variance

The aim of the present study was to evaluate the effect of replacement therapy onset on CNS and its relationship with IQ determinations. For this purpose we studied both neuropsychologic development and neurophysiologic changes (using LLSEP and event-related potentials) in $15 \mathrm{CH}$ patients, detected by neonatal screening, who began replacement therapy within the first 2 mo of life, and $11 \mathrm{CH}$ patients, clinically diagnosed in prescreening period, who started the replacement therapy much later.

\section{METHODS}

\section{Subjects}

Twenty-six children and adolescents (20 girls and six boys) with primary $\mathrm{CH}$ were studied. Excluded were patients showing symptoms of other primary neurologic or ophthalmologic diseases, patients with recurrent or acute otitis, and patients taking ototoxic drugs. $\mathrm{CH}$ patients born before term ( $<37 \mathrm{wk})$ or patients with perinatal problems (asphyxia, hyperbilirubinemia) were excluded. The $\mathrm{CH}$ patients were assigned to two groups: group A included 15 children (13 girls and two boys; mean age \pm SD: $9.93 \pm 0.81 \mathrm{y})$, detected by the screening 
program for $\mathrm{CH}$, covering infants born after 1980. Age, $\mathrm{T}_{4}$, and $\mathrm{TSH}$ concentrations at $\mathrm{CH}$ diagnosis are showed in Table 1. On the basis of thyroid imaging studies, eight patients were diagnosed to be athyreotic; four patients had ectopic and one had hypoplastic glands. The remaining two patients had familial dyshormonogenesis. Replacement therapy was started at $1.0 \pm$ $0.6 \mathrm{mo}$ : initial L-thyroxine dosage range was: $8-10 \mu \mathrm{g} / \mathrm{kg} / \mathrm{d}$. Group B consisted of 11 patients (seven girls and four boys; mean age $14.42 \pm 2.24 \mathrm{y}$ ), clinically diagnosed before the beginning of the $\mathrm{CH}$ screening program. Diagnosis was confirmed by the measurements of serum $\mathrm{T}_{4}$ and $\mathrm{TSH}$ concentrations (Table 1). Replacement therapy was started at 2-60 mo. Some patients were initially treated with thyroid USP. Initial doses of replacement therapy were similar to the ones of group A.

All patients were followed quarterly in our clinics and replacement therapy was adjusted to maintain peripheral thyroid hormones concentrations in the upper half of the ageappropriate range.

Twenty healthy children and adolescents (eight girls and 12 boys; $12.25 \pm 3.74 \mathrm{y}$ ) were studied as a control group for neurophysiologic examination. Inclusion criteria were: born at term ( $>37 \mathrm{wk}$ ), negative at the screening test for thyroid dysfunction; no history of brain insults (e.g. meningitis, convulsions, apnea), normal psychomotor development; no history or symptoms of recurrent or acute otitis; no ototoxic drugs.

Informed consent was obtained from all the parents and legal guardians of the $\mathrm{CH}$ patients and control subjects. The study was performed in accordance with the Declaration of Helsinki, as modified in 1983 .

\section{Procedure}

IQ was tested using the WISC-R (10). Briefly, the scale is made up of six verbal (information, similarities, arithmetic, vocabulary, comprehension, and digit span) and six performance subtests (picture completion, mazes, block design, picture arrangement, object assembly, digit symbol). Each subtest contributed equally to the total score. We selected two subtests in which the velocity of execution weights on the attribution of the score. Block design subtest points out the subjects' intellectual potentialities and is the expression of an organic integrity of the CNS. Digit symbol subtest measures the ability of visual-motor coordination, the velocity and the precision during the associative process, and above all the child's attention during the execution of the test.

Table 1. Age, $T_{4}$, and TSH concentrations at diagnosis in two groups of $\mathrm{CH}$ patients

\begin{tabular}{lccc}
\hline & Group A & Group B & Reference values \\
\hline Age $(m o)$ & $1.04 \pm 0.55$ & $9.96 \pm 17.09$ & \\
& $(0.4-2)$ & $(2-60)$ & \\
$\mathrm{T}_{4}($ nmol/L) & $49.5 \pm 22.7$ & $29.1 \pm 23.0$ & $63.4-145.7$ \\
& $(12.9-77.2)$ & $(6.5-82.4)$ & \\
TSH $(m U / L)$ & $207.6 \pm 195.1$ & $163.8 \pm 153.1$ & $0.2-4.0$ \\
& $(49.7-730)$ & $(20-320)$ & \\
\hline
\end{tabular}

Data are expressed as mean $\pm \mathrm{SD}$; the range of values is in parentheses.
Neurophysiologic evaluations were performed using the Nicolet Pathfinder Mega equipment (Nicolet Instrument Corp., Madison, WI).

Auditory P300. The P300 wave was evoked by auditory oddball paradigm. We recorded auditory potentials after infrequent high pitched $(2000 \mathrm{~Hz}, 80 \mathrm{~dB} \mathrm{nHL}$, target stimulus) and frequent low pitched $(1000 \mathrm{~Hz}, 70 \mathrm{~dB} \mathrm{nHL}$, non-target stimulus) tone bursts of $0.1 \mathrm{~ms}$ duration (with a 20 -ms plateau and 9.9-ms rise/fall time). The subjects were seated upright, and their visual attention was fixed at a marked point on the wall in front of them. They had to be attentive to the tone presented to both ears through headphones in a random sequence with a $20 \%$ probability of target stimulus (total stimuli 200 tone bursts). During the test, each subject was asked to count mentally only the target tones and to report the number at the end of each run. To be sure of a sustained level of attention, the test was replicated three times, and the total number of counted target tones had to be corrected for at least two full runs. Data were recorded from 16 electrodes attached to the scalp with collodion according to the 10-20 International System and referred to linked ears (11). Electrodes were placed laterally to each eye for monitoring the ocular blink (electro-oculogram). The records in which the EEG or electro-oculogram exceeded $\pm 90-\mathrm{mV}$ wave were rejected automatically. Separate averages were obtained for target and non-target stimulus tests. As described by Goodin et al. (12). the response to the frequent tone consists of a negative (N1), positive (P2) deflections; the response to the infrequent tone is more complex: negative $(\mathrm{N} 1)$, positive $(\mathrm{P} 2)$, negative $(\mathrm{N} 2)$, positive (P3) deflections. $\mathrm{N} 1$ and $\mathrm{P} 2$ latencies were identified in responses to frequent tones; $\mathrm{N} 2$ and $\mathrm{P} 3$ in response to rare tones. In cases in which the P3 peak was not well defined, lines were drawn parallel to the ascending and descending slopes of the P3 wave, and the intersection of these lines was taken as the P3 latency $(13,14)$. Amplitude was measured as peak to peak.

$\boldsymbol{L} \boldsymbol{L}$-SEP. LL-SEP were analyzed as previously described (8). Briefly, to minimize interferences, subjects laid comfortably on a bed, awake, and eyes open and fixing a point to avoid eye movements and drowsiness and to reduce excessive $\alpha$ activity in the EEG. Stimulations were applied to right and left median nerves at the wrist, with a random frequency of stimulation lower than $0.3 \mathrm{~Hz}$, stimulus duration of $0.1 \mathrm{~ms}$, and intensity just above motor twitch level. Analysis time was 600 $\mathrm{ms}$ with $60 \mathrm{~ms}$ of prestimulus time.

In addition, the topographical distribution of P300 either for auditory and LL-SEPs were analyzed using the maps measured for each subject at the peak of the maximum positive wave.

\section{Statistical Analysis}

IQ results are expressed as median (range), being a noncontinuous variable. Comparisons between groups were performed using the Mann-Whitney test.

Neurophysiologic variables were normally distributed. Data are expressed as mean $\pm \mathrm{SD}$. Differences between the three groups were assessed by one-way ANOVA. Further comparisons between groups were performed with the Scheffé post hoc 
test. Due to the age differences, analyses were also performed correcting for this confounding effect.

The relationships between variables were studied by calculating either the Pearson simple correlation coefficient (continuous variables) or the Spearman rank correlation coefficient (noncontinuous variables), and its significance as to a null hypothesis, $\rho=0$.

An unpaired $t$ test was used to establish significant differences of peak latencies and topographical distribution of evoked potentials between hypothyroid children and control subjects. $p$ value maps were reconstructed using $t$ values of the different electrodes. Values of latencies of the two study groups were considered abnormal when exceeding a $2.5 \mathrm{SD}$ of normal subjects. In each patient changes of topographical distribution were assessed using $z$-score values, and a value of 2.5 was chosen as the upper normal limit.

All significance tests were conducted at the $\alpha=0.05$ level and were two-tailed.

\section{RESULTS}

We did not find significant differences between the ages of the two groups of $\mathrm{CH}$ patients and control subjects $(p>0.05)$ (Fig. 1, $a$ and $b$ ). Ages of group A patients were significantly lower than those of group $\mathrm{B}(p<0.05)$.

Free $\mathrm{T}_{4}$ levels were within normal limits in all $\mathrm{CH}$ patients after 3 mo of replacement therapy. TSH concentrations fell

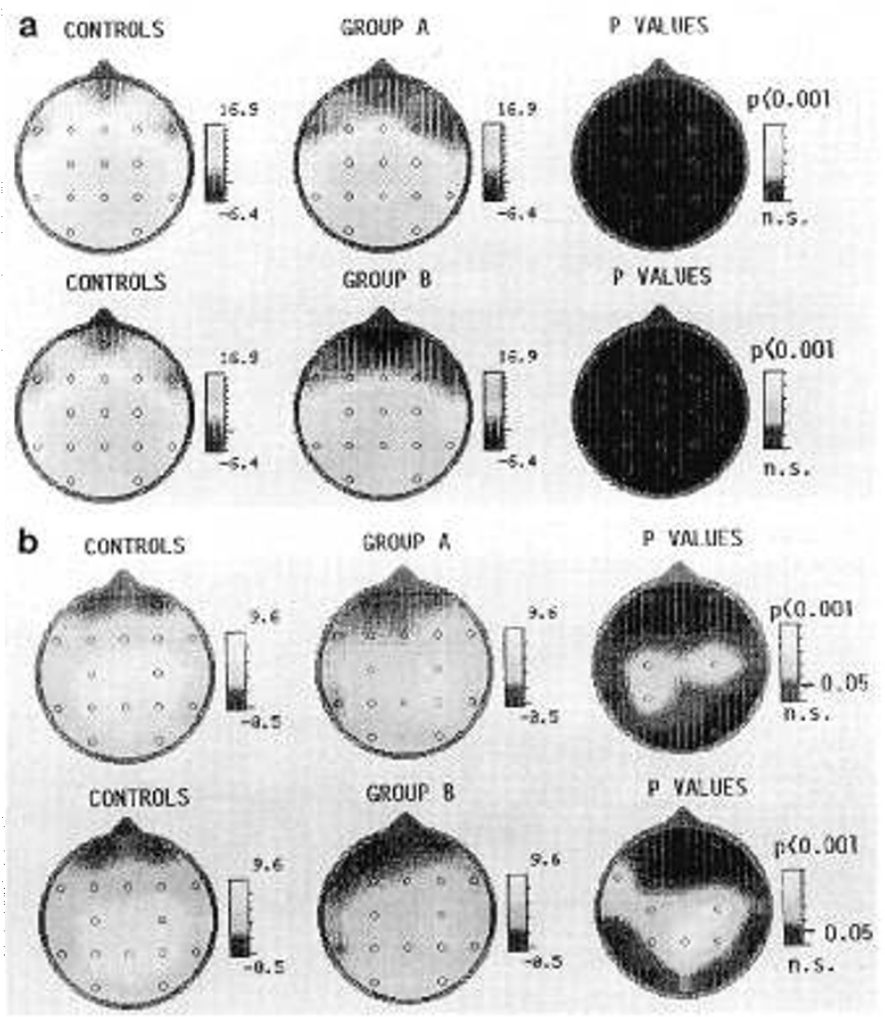

Figure 1. $a$, Topographic distribution of Auditory P300 amplitude in control and hypothyroid children. Right panel shows the statistical comparison. No significant differences were present for both groups of patients. $b$, Topographic of LL-SEP P300 amplitude in control and hypothyroid children. Significant differences were present in both groups, more pronounced in group B, more evident in centro-parietal areas. within normal range after 3 mo in 21 patients. In the remaining five patients TSH was within normal limits after 6 mo.

IQ. Median IQ values and ranges are shown in Table 2. Global and performance scores as well as block design and digit symbol subtests were significantly lower in group B than in group $\mathrm{A}$.

All patients showed reproducible Auditory P300 and LLSEP components. Frequencies of alterations for LL-SEP latencies were: $40 \%$ in group A and $64 \%$ in group B, whereas Auditory P300 was altered in 7 and 18\%, respectively (Table 3). Neurophysiologic studies detected CNS abnormalities in $82 \%$ of clinically diagnosed hypothyroid children (group B). All of these patients had low mental development indexes (median ID = 75.5). Surprisingly, $47 \%$ of the children detected by neonatal screening, having normal mental development index (median IQ = 109), showed at least one abnormal neurophysiologic test.

Auditory P300. Results of neurophysiologic tests are shown in Table 4. All patients showed normal P1 latencies values suggesting that primary auditory cortex and auditory association areas received normal projections. An example of Auditory P300 curves is shown in Figure 2. However, N1 wave latencies of auditory P300 were significantly different in the three groups (one-way ANOVA: $F=3.8 ; p=0.03$ ). A post hoc test showed a significant difference between groups $A$ and $\mathrm{B}(p<0.05)$. The difference was still present when differences in age were taken into account. N1 latency was inversely correlated with global IQ $\left(r_{s}=-0.50 ; p=0.03\right)$, performance $I Q\left(r_{s}=-0.49 ; p=0.04\right)$ and block design subtest $\left(r_{s}=\right.$ $-0.53 ; p=0.02$ ). We did not find significant differences in P3 wave latencies of auditory P300 among the study groups (one-way ANOVA: $F=1.2 ; p=0.31$ ). P3 latencies were inversely correlated with block design $\left(r_{s}=-0.49 ; p=0.02\right)$ and digit symbol $\left(r_{s}=-0.46 ; p=0.03\right)$ subtests. Neither N1 nor $\mathrm{P} 3$ waves showed significant correlations with $\mathrm{T}_{4}$ levels at diagnosis $(r=0.38 ; p=0.30$ and $r=0.25 ; p=0.32$, respectively) or age at onset of treatment $(r=0.03 ; p=0.89$ and $r=0.007 ; p=0.97$, respectively).

$\boldsymbol{L} L$-SEP. LL-SEP results are shown in Table 4. An example of LL-SEP curves is shown in Figure 3. P300 latencies of LL-SEP were significantly different in the three groups (oneway ANOVA: $F=9.4 ; p=0.0004)$. Post hoc comparisons showed significantly lower latencies in the control group,

Table 2. IQ scores determined using the WISC-R in two groups of CH patients

\begin{tabular}{lcccc}
\hline & \multicolumn{2}{c}{ Group A } & \multicolumn{2}{c}{ Group B } \\
\hline Global $\dagger$ & 110 & $(84-124)$ & 77 & $(69-94)$ \\
Performance tests $\dagger$ & 108 & $(84-130)$ & $73.5(67-93)$ \\
Block design $\ddagger$ & $11.5(5-16)$ & 8 & $(5-12)$ \\
Digit symbol $\ddagger$ & 8 & $(6-13)$ & $6 \quad(1-7)$ \\
\hline
\end{tabular}

Data are expressed as median (range). Comparison between groups were performed using the Mann-Whitney test. Normal IQ values are: global and performance tests $>80$; block design and digit symbol $>7$.

$\dagger \uparrow$ Significantly different.

$\dagger p \leq 0.001$.

$\ddagger p \leq 0.01$. 
Table 3. Frequencies of altered Auditory P300 and LL-SEP latencies and maps in two groups of $\mathrm{CH}$ children

\begin{tabular}{|c|c|c|c|}
\hline & Latencies & Maps & Both \\
\hline \multicolumn{4}{|c|}{ Auditory P300 } \\
\hline Group A & $1 / 15 \quad(6.7)^{a}$ & $1 / 15 \quad(6.7)$ & $1 / 15 \quad(6.7)$ \\
\hline Group B & $1 / 11$ & $1 / 11$ & $2 / 11$ \\
\hline \multicolumn{4}{|l|}{ LL-SEP } \\
\hline Group A & $4 / 15(26.6)$ & $3 / 14(21.4)$ & $6 / 15 \quad(40)$ \\
\hline Group B & $5 / 11(45.4)$ & $2 / 11(18.1)$ & $7 / 11(63.6)$ \\
\hline
\end{tabular}

${ }^{a}$ Values in parentheses are percent.

Table 4. Results of neurophysiologic tests in two groups of $\mathrm{CH}$ patients and control subjects

\begin{tabular}{|c|c|c|c|}
\hline & Group A & Group B & Controls \\
\hline$n$ & 15 & 11 & 20 \\
\hline \multicolumn{4}{|l|}{ Auditory P300 } \\
\hline $\mathrm{N} 1$ latency $(\mathrm{ms})$ & $108.2 \pm 21.6$ & $131.6 \pm 17.4 \dagger$ & $114.5 \pm 20.5$ \\
\hline P3 latency $(\mathrm{ms})$ & $310.9 \pm 21.7$ & $322.3 \pm 19.2$ & $313.0 \pm 18.0$ \\
\hline \multicolumn{4}{|l|}{ LL-SEP } \\
\hline P300 latency $(\mathrm{ms})$ & $339.9 \pm 38.5$ & $355.7 \pm 24.8$ & $310.2 \pm 23.1 \dagger$ \\
\hline $\begin{array}{l}\text { P40-P300 interpeak } \\
\quad(m s)\end{array}$ & $295.8 \pm 37.5$ & $308.7 \pm 26.3$ & $266.6 \pm 22.7+9$ \\
\hline
\end{tabular}

Data are expressed as mean $\pm \mathrm{SD}$.

$\dagger$ Significantly different from group A, Sheffé post hoc test; $p<0.05$.

$\ddagger$ Significantly different from group $\mathrm{B}$, Sheffé post hoc test; $p<0.01$.

I Significantly different from group B, Sheffé post hoc test; $p<0.05$.

compared with both groups of $\mathrm{CH}$ patients (Table 4). P300 latencies were inversely correlated with global IQ $\left(r_{s}=-0.43\right.$; $p=0.04)$ and performance IQ $\left(r_{s}=-0.44 ; p=0.04\right)$. No correlation was found with $\mathrm{T}_{4}$ levels at diagnosis of $\mathrm{CH}(r=$ $-0.27 ; p=0.27)$, nor with age at onset of treatment $(r=0.14$; $p=0.49)$. P40-P300 interpeaks were significantly different in the three groups (one-way ANOVA: $F=8.4 ; p=0.0009$ ). Interpeaks were significantly increased in $\mathrm{CH}$ patients (Table 4). Global IQ and performance IQ were weakly inversely correlated with P40-P300 interpeaks $\left(r_{s}=-0.39 ; p=0.06\right.$ and $r_{s}=-0.40 ; p=0.06$, respectively). No correlation was found with $\mathrm{T}_{4}$ levels at diagnosis of $\mathrm{CH}(r=0.28 ; p=0.24)$ nor with age at onset of treatment $(r=0.13 ; p=0.51)$.

The topographic distribution of the Auditory P300 was similar in the $\mathrm{CH}$ groups and in controls, and it showed the characteristic adult distribution centered in the centro-parietooccipital derivation (Fig. 1a). A significant abnormal topographic distribution of LL-SEP P300 wave was present in both groups, but more pronounced in group B (Fig. 1b).

\section{DISCUSSION}

Thyroid hormones are important for normal fetal brain development (15); in fact, they are known to regulate gliogenesis and myelinogenesis $(16,17)$. Moreover, thyroid hormones may act as a time clock, terminating proliferation and stimulating differentiation (15). Neonatal hypothyroidism results in an asynchrony of developmental events at a critical period of maturation and differentiation of the developing nervous system.

Neonatal screening programs has improved the prognosis for mental development of $\mathrm{CH}$ children (1-6). Slight developmental delay related to signs of prenatal and perinatal hypothyroid-
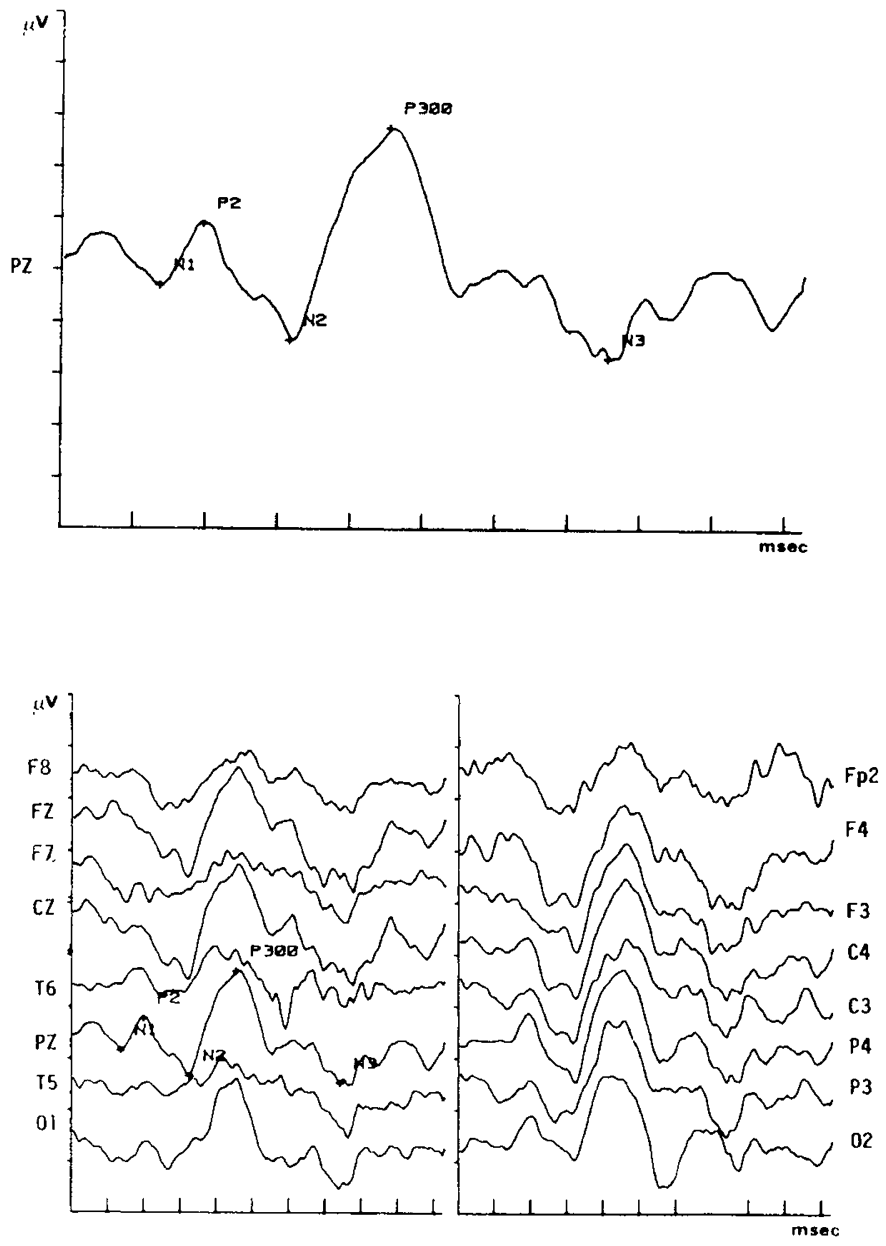

Figure 2. Auditory $\mathbf{P} 300$ curves in a control subject. The top graph illustrates the mean curve. The bottom graph shows the Auditory P300 recorded by 16 electrodes.

ism (retarded skeletal maturation at the time of diagnosis, low initial serum thyroxine, and athyreosis) has also been reported in about $15 \%$ of screened $\mathrm{CH}$ patients $(1,3,6)$. Despite early treatment, some $\mathrm{CH}$ children with normal IQ may show psychomotor abnormalities, such as impairment of balance, fine motor disorders, and learning difficulties, suggesting underlying problems of the CNS $(1,5,7)$.

We studied neuropsychologic development and neurophysiologic changes in $\mathrm{CH}$ patients detected by neonatal screening, who began the replacement therapy within the first 2 mo of life, and $\mathrm{CH}$ patients clinically diagnosed in prescreening period, who started the replacement therapy much later. We measured IQ in $\mathrm{CH}$ patients with the WISC-R scale. As expected, nonscreened patients had lower IQ values than screened patients. The difference was comparably remarkable when the performance subtests were taken into account. We were interested in knowing whether lower IQ values were correlated to organic impairments of the CNS. For this purpose we measured two neurophysiologic indices: Auditory P300, which evaluates the attention and information processing, and LLSEP, which assess the integrity of sensory processing within the brain. The results of neurophysiologic studies confirmed the presence of a relevant CNS damage in patients of group B: in fact, we found a significant delay of N1 wave of Auditory 

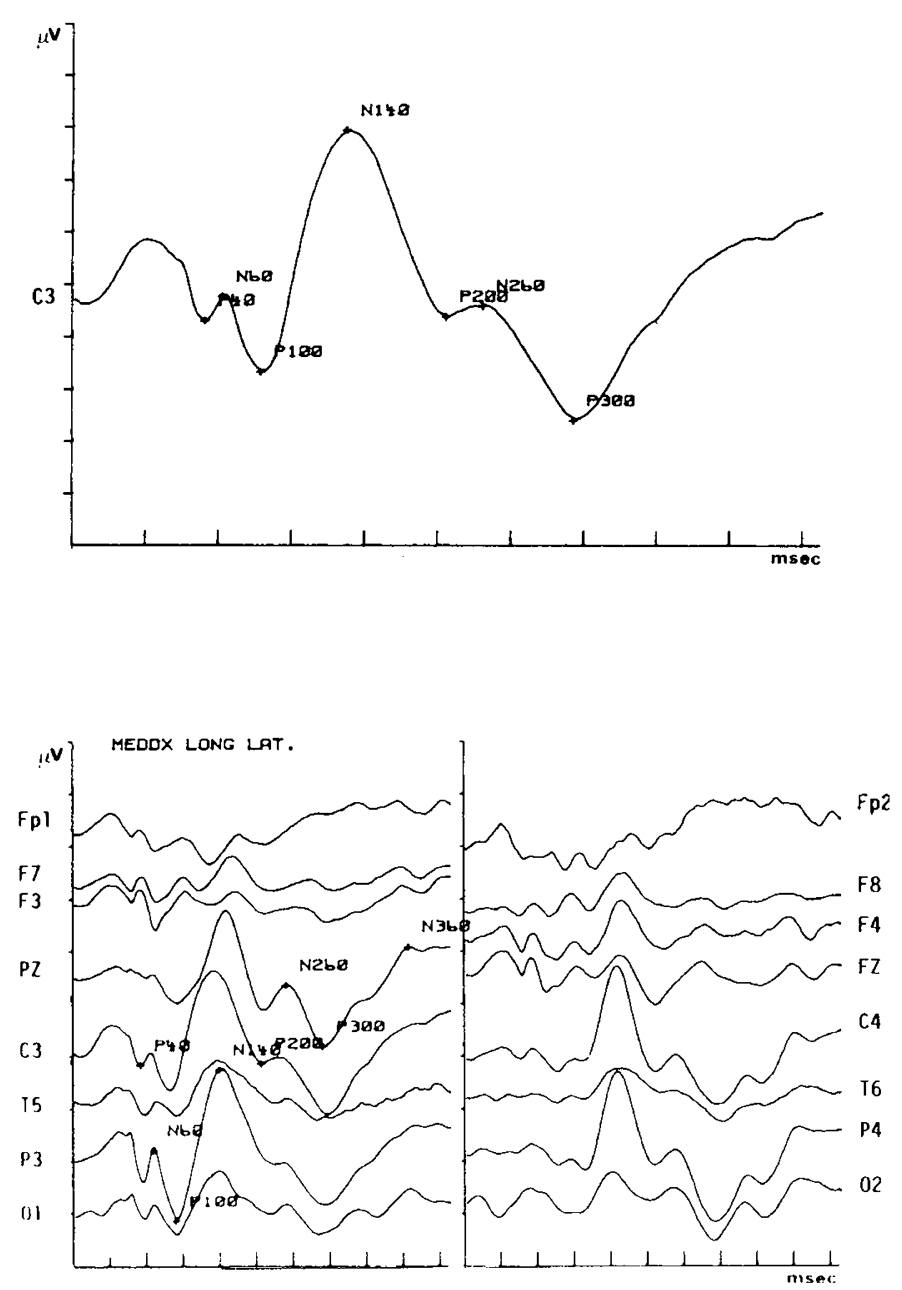

Figure 3. LL-SEP curves in a control subject. The top graph illustrates the mean curve. The bottom graph shows the LL-SEP recorded by 16 electrodes.

P300, significant increases of P300 and P40-P300 LL-SEP interpeak latencies and remarkable alterations of topographic distributions of LL-SEP, compared with controls. These results confirm the presence of an attention deficit and a delayed nervous conduction velocity probably due by a delayed myelination process and the consequent desyncronization of sensitive input. Surprisingly, $47 \%$ of the children detected by neonatal screening, having normal mental development index (median IQ = 109), showed at least one abnormal neurophysiologic test. The finding could be due to the relatively delayed beginning of therapy in screened patients. The reason was that the patients we describe in the present study were among the first screened newborns in Italy. The screening program became effective in early 1980 , but the results of the test were not always available in reasonable time. Moreover, we did not measure minor motor impairment or behavior problems that could be present in these children.
We found inverse correlations between global and performance IQ and both neurophysiologic tests. The psychomotor abnormalities most frequently found even in $\mathrm{CH}$ patients treated from early life are clumsiness, fine motor disorders, and attention deficit. We, thus, selected the block design and digit symbol subtests which better reflect motor skills. Inverse correlations between the subtests and the N1 latency of the auditory P300 wave, related with attention, were found in our patients. These correlations suggest a subtle organic damage of CNS. Our data do not indicate whether it was the result of a prenatal event or whether other postnatal factors (such as psychosocial environment or the relative late onset of replacement therapy) played an important role. A study with a greater number of patients, homogeneous for the type of $\mathrm{CH}$ and onset of treatment, is needed to define this problem. The combined neurophysiologic tests are an adequate tool for longitudinal studies to discriminate subclinical organic neurologic involvement.

\section{REFERENCES}

1. Glorieux J, Dussault JH, Morisette J, Desjardins M, Letarte J, Guyda H 1985 Follow up at ages 5 and 7 years on mental development in children with hypothyroidism detected by the Quebec screening program. J Pediatr 107:913--915

2. Rovet J, Ehrich R, Sorbara D 1987 Intellectual outcome in children with fetal hypothyroidism. J Pediatr 110:700-704

3. Glorieux J, Desjardins M, Letarte J, Morissette J, Dussault JH 1988 Useful parameters to predict the eventual mental outcome of hypothyroid children. Pediatr Res 24:6-8

4. Heyerdahl S, Kase BF, Lie SO 1991 Intellectual development in children with congenital hypothyroidism in relation to recommended thyroxine treatment. J Pediatr 118:850-857

5. Fuggle PW, Grant DB, Smith I, Murphy G 1991 Intelligence, motor skills and behaviour at 5 years in early-treated congenital hypothyroidism. Eur J Pediatr 150:570-574

6. Glorieux J, Dussault J, Van Vliet G 1992 Intellectual development at age 12 years of children with congenital hypothyroidism diagnosed by neonatal screening. J Pediatr 121:581-584

7. MacFaul R, Dorner S, Brett EM, Grant DB 1978 Neurological abnormalities in patients treated for hypothyroidism from early life. Arch Dis Child 53:611-619

8. Colon EJ, Comi G 1990 Long latency somatosensory evoked potentials. In: Colon EJ, Visser SL (eds) Evoked Potentials Manual. Kluwer Academic Publishers, Amsterdam, The Nederlands, pp 279-306

9. Bongers-Schokking CJ, Colon EJ, Hoogland RA, de Groot CJ, Van den Brande JL 1991 Somatosensory evoked potentials in neonates with primary congenital hypothyroidism during the first week of therapy. Pediatr Res 30:34-39

10. Wechsler D 1974 Wechsler Intelligence Scale for Children-Revised. The Psychological Corp, New York

11. Jaspe HH 1958 The ten-twenty electrode system. Electroenceph Clin Neurophysiol $10: 371-375$

12. Goodin DS, Squires KC, Henderson BH, Starr A 1978 An early event-related cortical potential. Psychophysiology 15:360-365

13. Goodin DS, Squires KC, Handerson B, Starr A 1978 Long-latency event-related components of auditory evoked potentials in dementia. Brain 44:447-458

14. Brown SB, Marsh JT, La Rue A 1983 Experimental electrophysiological aging: P3 latency. EEG Clin Neurophysiol 55:277-285

15. Porterfield SP, Hendrich CE 1993 The role of thyroid hormones in prenatal and neonatal neurological development. Current perspectives. Endocr Rev 14:94-106

16. Tsujimura R, Karijama N, Hatotani N 1971 Disturbances of myelination in neonatally thyroidectomized rat brains. In: Lissak K (ed) Hormones and Brain Function. Plenum Press, New York, pp 69-78

17. Koper JW, Hoeben RC, Hochstenbach PMH, van Golde LMG, Lopes Cardozo M 1986 Effects of triiodothyronine on the synthesis of sulfolipidis by oligodendrocyteenriched glial cultures. Biochim Biophys Acta 887:327-334 